\title{
Über den Einfluß von Prednisolon auf die Empfindlichkeit von Strumagewebekulturen gegenüber dem Poliovirus Typ III (Saukett)
}

\author{
Von H. HöveL \\ Aus der Staatlichen Anstalt für experimentelle Therapie „Paul-Ehrlich-Institut“ \\ und dem Ferdinand-Blum-Institut für experimentelle Biologie zu Frankfurt am Main \\ (Kommissarischer Direktor: Prof. Dr. G. Heymans) \\ (Z. Naturforschg. 18 b, 374-377 [1963]; eingegangen am 21. September 1962)
}

\begin{abstract}
Verglichen wurde die Empfindlichkeit von gleichzeitig angelegten und gleichartig mit bzw. ohne Prednisolonsuccinat vorbehandelten Strumagewebekulturen und Affennieren-Gewebekulturen sowohl in $\mathrm{F}$ e r n b a $\mathrm{ch}$ - Kolben als auch in Vierkantflaschen gegenüber der Infektion mit gleichgroßen Dosen von Poliovirus Typ III (Saukett).

Hinsichtlich der Ausbildung eines mikroskopisch ablesbaren cytopathologischen Effektes nach Beimpfung mit diesem Poliovirus ließen sich die Strumagewebekulturen durch 0,125 und $0,250 \mathrm{mg}$ Prednisolon pro $\mathrm{cm}^{3}$ Nährmedium verbessern, während unter den Affennieren-Gewebekulturen nur ein hemmender Effekt bei hohen Prednisolon-Konzentrationen erkennbar war.

Die durch Virustitrationen erzielten Versuchsergebnisse zeigen, daß die Strumagewebekulturen in ihrer Empfindlichkeit gegenüber Typ III des Poliovirus durch einen Prednisolonzusatz von $0,125 \mathrm{mg} / \mathrm{cm}^{3}$ Nährmedium 6-8 Stdn. vor der Beimpfung optimal gesteigert werden konnten, während bei Affennieren-Gewebekulturen dieser Effekt ausblieb.

Weiterhin ist daraus zu ersehen, daß höhere Konzentrationen von Prednisolon sowohl bei Strumagewebekulturen als auch bei Affennieren-Gewebekulturen keine Steigerung der Empfindlichkeit zur Folge haben, sondern sich im Gegenteil hemmend auf die Virusvermehrung auswirken können.
\end{abstract}

Die Brauchbarkeit von Strumagewebekulturen (SGK) für Versuche mit Polioviren ist schon in früheren Arbeiten auf verschiedene Art und Weise untersucht worden ${ }^{1,2,4,5 ; 7}$. Es hatte sich dabei herausgestellt, daß die Empfindlichkeit der SGK gegenüber den Polioviren aller Typen im Vergleich zur Affennieren-Gewebekultur (AGK) geringer ist. Bei Verwendung des Typ III war der Unterschied zwischen den beiden Gewebekulturarten besonders deutlich, was uns veranlaßte, nach Möglichkeiten zur Steigerung der Empfindlichkeit der SGK besonders gegenüber diesem Poliovirustyp zu suchen.

Orientierende Vorversuche ließen erkennen, daß ein bestimmter Zusatz von Prednisolonsuccinat zum sonst üblichen Nährmedium eine Empfindlichkeitssteigerung der SGK gegenüber Poliovirus Typ III zur Folge hat.

Über vergleichende Empfindlichkeitsprüfungen von SGK einerseits und AGK andererseits gegenüber diesem Virus bei verschiedener PrednisolonKonzentration im Nährmedium soll hier berichtet werden.

1 H. Finger u. H. Hövel, Zbl. Bakteriol., Parasitenkunde, Infektionskrankh. Hyg., I. Abt. Orig. 184, 426 [1962].

2 H. Finger u. H. Hövel, Naturwissenschaften 20, 649 [1961].

${ }^{4}$ H. Hövel u. W. Schneider, Zbl. Bakteriol., Parasitenkunde, Infektionskrankh. Hyg., I. Abt. Orig. 177, 285 [1960.]
Für die Anzucht der Versuchskulturen wurden bei Operationen gewonnene Strumagewebeteile bzw. Rhesusaffen-Nieren verarbeitet ${ }^{4,7}$. Das Zellsediment wurde im Verhältnis $1: 800$ in einer mit Natriumbicarbonat auf ein $p_{\mathrm{H}}$ von $6,8-7,0$ abgestimmten, 0,5-proz. Lösung von Lactalbuminhydrolysat in $\mathrm{H}$ a n k s-Lösung mit Zusatz von 5\% Kälberserum und NSP (Neomycin, Streptomycin und Penicillin je $0,1 \mathrm{mg} / \mathrm{cm}^{3}$ Nährmedium) aufgeschwemmt. Die Aussaat erfolgte in F e r n b a ch Kolben $\left(100 \mathrm{~cm}^{3}\right.$ Zellsuspension) und Vierkantflaschen $\left(15 \mathrm{~cm}^{3}\right.$ Zellsuspension), die im Brutraum bei $37^{\circ} \mathrm{C}$ aufgestellt wurden. Nach 3 bis 4 Tagen war die Haftung der Zellen an der Gefäßwand soweit fortgeschritten, daß das Medium gewechselt werden konnte. Die Kulturen erhielten nun TCM 199 mit 5\% Kälberserum und $0,1 \mathrm{mg} / \mathrm{cm}^{3}$ Leukomycin (Bayer), abgestimmt auf $p_{\mathrm{H}} 7,2$. Etwa 1 Woche nach der Aussaat waren die Kulturen zu einem geschlossenen Zellrasen ausgewachsen und konnten in den Versuch genommen werden.

Die betreffenden SGK und AGK wurden erneut mit frischem Medium gefüttert. Dieses bestand diesmal aus TCM mit NSP ohne Serumzusatz, wieder abgestimmt auf ein $p_{\mathrm{H}}$ von 7,2 . Dazu kam für je eine Kulturflasche mit SGK und AGK ein Zusatz von $0,125 \mathrm{mg}, 0,250$ oder $0,5 \mathrm{mg}$ Prednisolonsuccinat (Decortin-H, Merck) pro $\mathrm{cm}^{3}$ Nährmedium. Die Kontrollkulturen für beide Gewebearten blieben jedesmal ohne diese Zusätze.

j R. J. V. Pulvertaft, J. R. Davies, L. Weiss u. J. H. WilKinson, J. Pathol. Bacteriol. 77, 19 [1959].

7 W. Schneider, H. Hövel u. J. Krüger, Arb. Paul-EhrlichInst. 56, 55 [1961]. 
Nach 6-8 Stdn. Brutraumaufstellung wurden alle auf diese Art und Weise vorbehandelten Vergleichskulturen mit $0,5 \mathrm{~cm}^{3}$ einer etwa $10 \mathrm{dim}$ (dosis infectiosa media) in $0,5 \mathrm{~cm}^{3}$ enthaltenden Verdünnung von fortlaufend auf AGK gezüchtetem Poliovirus Typ III (Saukett) infiziert.

Zwecks Beobachtung der zu erwartenden cytopathologischen Effekte (CPE) sind alle Versuchskulturen ab 3. Tag nach der Beimpfung täglich mikroskopiert worden.

Um die Größe der Virusvermehrung in den einzelnen Kulturen feststellen und dann vergleichen zu können, erfolgte durchschnittlich 1 Woche nach der Infektion die Paralleltitration der durch Tiefkühlung aufgeschlossenen Zellmediumgemische auf AGK in Rollrandröhrchen. Pro Verdünnungsstufe wurden für die Abzüge aus F e rnbach-Kolben 10 Röhrchen und für solche aus Vierkantflaschen 5 Röhrchen eingesetzt. Die Berechnung des Virustiters erfolgte nach der Flächenmethode (v. D. W AERDEN).

Verglichen wurde sowohl der Zeitpunkt des Auftretens von CPE als auch das Ergebnis der Virustitrationen zwischen SGK und AGK, zwischen den verschiedenen Prednisolon-Konzentrationen innerhalb der gleichen Gewebeart und zwischen F e r n b a c h-Kolben- und Vierkantflaschen-Kulturen. Die letztgenannte Gegenüberstellung erklärt sich aus der verschieden großen Verdünnung der für alle Kulturen gleichgroßen Infektionsdosis durch die unterschiedlichen Mediummengen in den betr. Kulturgefäßen.

Das erste sichtbare Auftreten eines CPE konnte durchschnittlich bei den SGK am 5.-6., bei den AGK am 4. - 5. Tag nach der Infektion beobachtet werden.

Unter den SGK betraf dies vorwiegend die mit 0,125 und $0,250 \mathrm{mg}$ Prednisolon pro $\mathrm{cm}^{3} \mathrm{Nähr}$ medium gefütterten Kulturen, während die mit $0,5 \mathrm{mg} / \mathrm{cm}^{3}$ oder ohne Prednisolon vorbehandelten erst später oder manchmal gar keine CPE zeigten.

Die AGK ließen fast keine Unterschiede untereinander erkennen mit Ausnahme derjenigen, die einen Prednisolonzusatz von $0,5 \mathrm{mg} / \mathrm{cm}^{3}$ erhalten hatten. Diese entwickelten erst spät auftretende und langsam fortschreitende CPE.

Zwischen Fernbach-Kolben- und Vierkantflaschen-Kulturen waren hinsichtlich des Auftretens eines CPE keine nennenswerten Unterschiede festzustellen.

Wie oben beschrieben, wurde etwa eine Woche nach Versuchsbeginn zwecks Feststellung der Virusvermehrung jeder Kulturflascheninhalt titriert. Die

\begin{tabular}{|c|c|c|c|c|c|c|c|c|c|c|c|c|}
\hline \multirow{2}{*}{$\begin{array}{l}\quad \text { Prednisolon: } \\
\text { (pro ccm } \\
\text { Nährmedium) }\end{array}$} & \multicolumn{3}{|c|}{ - Ohne - } & \multicolumn{3}{|c|}{$0,125 \mathrm{mg}$} & \multicolumn{3}{|c|}{$0,250 \mathrm{mg}$} & \multicolumn{3}{|c|}{$0,5 \mathrm{mg}$} \\
\hline & SGK & AGK & Diff. & SGK & AGK & Diff. & SGK & AGK & Diff. & SGK & AGK & Diff. \\
\hline & $\begin{array}{l}6,2 \\
4,9 \\
4,5 \\
6,3 \\
7,2\end{array}$ & $\begin{array}{l}7,4 \\
8,4 \\
7,9 \\
7,4 \\
8,4\end{array}$ & $\begin{array}{l}1,2 \\
3,5 \\
3,4 \\
1,1 \\
1,2\end{array}$ & $\begin{array}{l}7,9 \\
4,9 \\
7,2 \\
6,6 \\
7,3\end{array}$ & $\begin{array}{l}8,3 \\
8,0 \\
7,7 \\
7,3 \\
7,6\end{array}$ & $\begin{array}{l}0,4 \\
3,1 \\
0,5 \\
0,7 \\
0,3\end{array}$ & $\begin{array}{l}6,9 \\
7,5 \\
7,2 \\
6,5 \\
5,4\end{array}$ & $\begin{array}{l}7,5 \\
8,3 \\
7,9 \\
6,9 \\
8,4\end{array}$ & $\begin{array}{l}0,6 \\
0,8 \\
0,7 \\
0,4 \\
3,0\end{array}$ & $\begin{array}{l}6,4 \\
7,5 \\
3,7 \\
6,3 \\
5,5\end{array}$ & $\begin{array}{l}7,9 \\
8,0 \\
8,3 \\
7,8 \\
7,6\end{array}$ & $\begin{array}{l}1,5 \\
0,5 \\
4,6 \\
1,5 \\
2,1\end{array}$ \\
\hline $\begin{array}{l}\text { Summe } \\
\text { Mittel }\end{array}$ & $\begin{array}{c}29,1 \\
5,82\end{array}$ & $\begin{array}{c}39,5 \\
7,90\end{array}$ & $\begin{array}{c}10,4 \\
2,08\end{array}$ & $\begin{array}{r}33,9 \\
6,78\end{array}$ & $\begin{array}{c}38,9 \\
7,78\end{array}$ & $\begin{array}{l}5,0 \\
1,00\end{array}$ & $\begin{array}{c}33,5 \\
6,70\end{array}$ & $\begin{array}{c}39,0 \\
7,80\end{array}$ & $\begin{array}{l}5,5 \\
1,10\end{array}$ & $\begin{array}{c}29,4 \\
5,88\end{array}$ & $\begin{array}{c}39,6 \\
7,92\end{array}$ & $\begin{array}{c}10,2 \\
2,04\end{array}$ \\
\hline $\begin{array}{l}\text { Mittl. Fehler } \\
\text { d. mittl. Diff. }\end{array}$ & & & 0,56 & & & 0,53 & & & 0,48 & & & 0,69 \\
\hline
\end{tabular}

F e r n b a c h - Kolben.

\begin{tabular}{|c|c|c|c|c|c|c|c|c|c|c|c|c|}
\hline \multirow{2}{*}{$\begin{array}{l}\quad \text { Prednisolon: } \\
\text { (pro ccm } \\
\text { Nährmedium) }\end{array}$} & \multicolumn{3}{|c|}{ - Ohne - } & \multicolumn{3}{|c|}{$0,125 \mathrm{mg}$} & \multicolumn{3}{|c|}{$0,250 \mathrm{mg}$} & \multicolumn{3}{|c|}{$0,5 \mathrm{mg}$} \\
\hline & SGK & AGK & Diff. & SGK & AGK & Diff. & SGK & AGK & Diff. & SGK & AGK & Diff. \\
\hline & $\begin{array}{l}5,7 \\
5,3 \\
5,7 \\
7,7 \\
4,3\end{array}$ & $\begin{array}{l}7,9 \\
8,1 \\
7,3 \\
8,3 \\
7,1\end{array}$ & $\begin{array}{l}2,2 \\
2,8 \\
1,6 \\
0,6 \\
2,8\end{array}$ & $\begin{array}{l}5,9 \\
6,7 \\
7,1 \\
8,1 \\
7,1\end{array}$ & $\begin{array}{l}7,9 \\
7,9 \\
7,3 \\
8,1 \\
7,3\end{array}$ & $\begin{array}{l}2,0 \\
1,2 \\
0,2 \\
0 \\
0,2\end{array}$ & $\begin{array}{l}4,3 \\
3,9 \\
7,1 \\
7,3 \\
6,9\end{array}$ & $\begin{array}{l}7,9 \\
7,9 \\
7,5 \\
6,7 \\
7,5\end{array}$ & $\begin{array}{r}3,6 \\
4,0 \\
0,4 \\
-0,6 \\
0,6\end{array}$ & $\begin{array}{l}5,3 \\
4,3 \\
6,7 \\
5,5 \\
5,9\end{array}$ & $\begin{array}{l}7,9 \\
6,1 \\
7,3 \\
8,3 \\
7,5\end{array}$ & $\begin{array}{l}2,6 \\
1,8 \\
0,6 \\
2,8 \\
1,6\end{array}$ \\
\hline $\begin{array}{l}\text { Summe } \\
\text { Mittel }\end{array}$ & $\begin{array}{r}28,7 \\
5,74\end{array}$ & $\begin{array}{c}38,7 \\
7,74\end{array}$ & $\begin{array}{r}10,0 \\
2,0\end{array}$ & $\begin{array}{c}34,9 \\
6,98\end{array}$ & $\begin{array}{c}38,5 \\
7,70\end{array}$ & $\begin{array}{l}3,6 \\
0,72\end{array}$ & $\begin{array}{c}29,5 \\
5,90\end{array}$ & $\begin{array}{r}37,5 \\
7,50\end{array}$ & $\begin{array}{l}8,0 \\
1,60\end{array}$ & $\begin{array}{l}27,7 \\
5,54\end{array}$ & $\begin{array}{c}37,1 \\
7,42\end{array}$ & $\begin{array}{l}9,4 \\
1,88\end{array}$ \\
\hline $\begin{array}{l}\text { Mittl. Fehler } \\
\text { d. mittl. Diff. }\end{array}$ & & & 0,41 & & & 0,38 & & & 0,92 & & & 0,39 \\
\hline
\end{tabular}


Ergebnisse sind aus der Tabelle (Abb. 1) und der Kurvendarstellung (Abb. 2) zu ersehen. An Stelle der Titer (Anzahl der dim pro $\mathrm{cm}^{3}$ ) wird jeweils der Logarithmus zur Basis 10 angegeben.

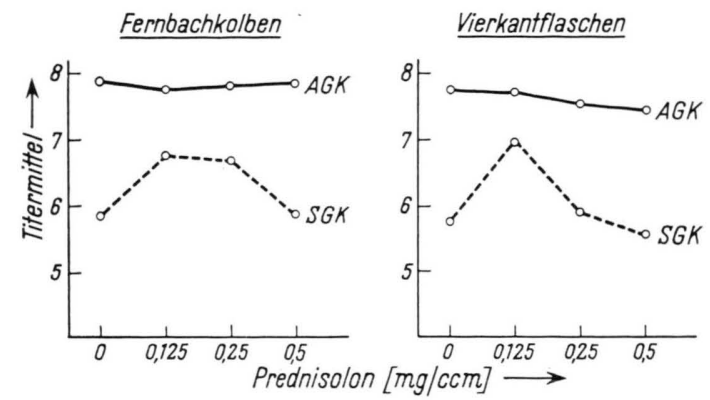

Abb. 2. Ergebnisse von Abb. 1 in Kurven.

Der Einfluß der jeweiligen Prednisolon-Konzentration im Nährmedium auf die Virusvermehrung läßt sich sowohl an Hand der Titermittel als auch durch den Vergleich der Mittel der einzelnen Titerdifferenzen zwischen SGK und AGK nachweisen.

Diese betragen, wie aus der Tabelle in Abb. 1 zu ersehen ist, für die vier Stufen der Prednisolon-Konzentration bei F e rn b a c h - Kolbenkulturen: 2,08, $1,0,1,1$ und 2,04 und bei Vierkantflaschen-Kulturen: 2,0, 0,72, 1,6 und 1,88. Die Darstellung der für die einzelnen Prednisolonzusätze errechneten Titermittel in Abb. 2 läßt sowohl das Zustandekommen dieser mittleren Titerdifferenzen als auch die Unterschiede zwischen den Gewebearten, den abgestuften Prednisolon-Konzentrationen und den Kulturgefäßarten leicht überbiicken.

Während die parallelgelaufenen AGK in F e r n $\mathrm{b}$ a ch-Kolben trotz verschiedener Prednisolonzusätze ziemlich gleichbleibende Titermittel ergaben, zeigten die mit 0,125 und $0,250 \mathrm{mg}$ Prednisolon pro $\mathrm{cm}^{3}$ Nährmedium vorbehandelten SGK in $\mathrm{F}$ e r n b a c h - Kolben eine Verbesserung ihrer Titermittel um 0,96 bzw. 0,88 gegenüber den prednisolonfreien Kontrollen. Bei den SGK mit $0,5 \mathrm{mg}$ Prednisolonzusatz pro $\mathrm{cm}^{3}$ sanken die Werte wieder bis fast auf diejenigen der Kontrollkulturen. Dies entspricht dem Absinken bzw. Ansteigen der Mittel der einzelnen Titerdifferenzen dieser F e r n b a c h - Kolben-Kulturen.

Die SGK in Vierkantflaschen erzielten gegenüber den Kontrollen eine noch deutlichere Steigerung des

8 M. M. Sigel u. A. R. Beasley, Proc. Soc. exp. Biol. Med. 88, 86 [1955].

9 R. B. Stewart, J. Bacteriol. 80, 25 [1960].

3 A. D. Greenberg u. R. B. Stewart, Proc. Soc. exp. Biol. Med. 106, 666 [1961].
Titermittels um 1,24 bei einem Prednisolonzusatz von $0,125 \mathrm{mg}$ pro $\mathrm{cm}^{3}$ Nährmedium. Bei den höheren Konzentrationen war dagegen ein zunehmender Rückgang der Werte bis unter diejenigen der Kontrollen festzustellen, was wiederum der Ab- bzw. Zunahme der mittleren Titerdifferenzen bei diesen Vierkantflaschen-Kulturen entspricht. Von den AGK in Vierkantflaschen zeigten alle Titermittel eine leicht fallende Tendenz bei steigender Prednisolonkonzentration.

Sigel und Beasley ${ }^{8}$ beobachteten gegensätzliche Effekte im Auftreten cytopathologischer Veränderungen durch Polioviren (Typ I und II) und Vacciniaviren auf HeLa-Zellkulturen bei Zusatz von 0,25 mg Cortison (Dehydrohydroxycorticosteronacetat) pro ml Medium. Eine Verzögerung und teilweise Hemmung trat auf, wenn Cortison gleichzeitig mit dem Virus auf die Kultur gebracht worden war. Eine Beschleunigung zeigte sich bei Cortisonzusatz zum Nährmedium schon während der Anzucht der Kulturen.

STewart ${ }^{9}$ erzielte mittels Cortisonacetat-Suspension $(0,1 \mathrm{mg} / \mathrm{ml}, 24 \mathrm{Stdn}$. vor der Beimpfung zugesetzt) höhere Titer von Psittacosevirus auf L-Zellen im Vergleich zu Kontrollkulturen ohne Cortison. Als mögliche Ursache wird ein Schutzeffekt des Cortisons auf die Kulturzellen angegeben. Außerdem wurde eine Verzögerung der Freigabe von Virus aus den Kulturzellen durch Cortisonzusatz beobachtet.

Greenberg und Stewart ${ }^{3}$ beobachteten bei mit $0,2 \mathrm{mg} / \mathrm{ml}$ Hydrocortison vorbehandelten HeLa-Zellen gegenüber Kulturen ohne Cortisonzusatz einen leichten Rückgang der Glucoseaufnahme und -speicherung. Nach Beimpfung mit Herpesvirus wurde dagegen eine vermehrte Glucoseaufnahme und -speicherung im Vergleich mit infizierten Kontrollkulturen ohne Cortison festgestellt.

Wellington und Moon ${ }^{10}$ sahen eine Hemmung des Zellwachstums bei Hydrocortison-Zusatz zu synthetischen Medien proportional zur Konzentration (50, 100, $200 \gamma / \mathrm{ml})$ und Einwirkungsdauer (24, 48,72 Stdn.).

Rohde, Schreiner und Vogel ${ }^{6}$ untersuchten die Reaktion von Fibroblasten-Mischkulturen auf den

10 J. S. Wellington u. H. D. Moon, Proc. Soc. exp. Biol. Med. 107, 556 [1961].

${ }^{6}$ B. Rohde, H. E. Schreiner u. A. Vogel, Arzneimittelforsch. 11, 392 [1961]. 
Zusatz von Corticoiden in verschiedener Konzentration. Cortison und Prednisolon zeigten einen Wirkungsumschlag von Wachstumshemmung bei höherer Dosierung zu deutlicher Wachstumsbeschleunigung bei niedriger Konzentration im Nährmedium. Hexadecadron förderte vorübergehend in allen Konzentrationen das Wachstum. ACTH-Zusatz zeigte nur bei niedrigen Konzentrationen ein mehr oder minder gutes Wachstum. Hohe Dosen lösten die Kulturzellen langsam auf.

Alle diese Beobachtungen, die bei Versuchen mit cortisonhaltigen Nährmedium auf wachsenden bzw. mit Virus infizierten Zellkulturen gemacht wurden, deuten darauf hin, daß eine direkte Beeinflussung des normalen und des durch Virusvermehrung veränderten Stoffwechsels von Kulturzellen durch Cortisonzusatz möglich ist. Dabei sind hinsichtlich der Auswirkung jedoch Dosierung und Zeitpunkt der Anwendung zu beachten.

Frau M. Reiche, Frau H. Troschier und Frau E. Kunn danke ich für sorgfältige technische Mitarbeit. Herr Diplom-Mathemathiker J. KR ̈ÜGER hat mich freundlicherweise bei der Auswertung der Versuchsergebnisse unterstützt.

\title{
The Components of Measles Virus and their Relation to Rinderpest and Distemper
}

\author{
By A. P. Waterson * and Rudolf Rott \\ Max-Planck-Institut für Virusforschung, Tübingen \\ and Gisela Ruckle-Enders \\ Hygiene-Institut der Universität Marburg \\ (Z. Naturforschg. 18 b, 377-384 [1963] ; eingegangen am 18. Februar 1963)
}

\begin{abstract}
Measles virus has been disintegrated by treatment with ether and Tween 80 . This destroys the infectivity, and physically disintegrates the particle, with the release of an inner component structurally like the nucleoprotein of Newcastle disease virus (NDV), and another structure similar to the haemagglutinin (HA) of NDV. The preparation after ether-tween treatment has an enhanced $\mathrm{HA}$ activity. The two components are separable in a $\mathrm{CsCl}$ density gradient. The inner component is probably a nucleoprotein. The HA component could be adsorbed by monkey erythrocytes but not eluted from them. The action on it of sodium metaperiodate suggests that a carbohydrate may be involved. The ether-tween preparation could be used as an antigen in the CF-reaction with antisera to rinderpest and distemper. It could also be used in the HA-inhibition test as a sensitive indicator with antisera to rinderpest and distemper, as well as to measles, giving higher titres in the HA. inhibition test than when the untreated virus was used. Injection of the ether-tween preparation stimulated the production of neutralizing, HA-inhibiting and complement-fixing antibodies in the rabbit.
\end{abstract}

The particle of measles virus has the same fine structure, as seen in the electron microscope, as that of the larger myxoviruses, i. e. the Newcastle diseasemumps-parainfluenza group ${ }^{1}$. When treated with a detergent (Triton X 100) ${ }^{1}$ structures similar to the haemagglutinin (HA) of Newcastle disease virus ${ }^{2}$ (NDV) are released. Measles virus has been shown to agglutinate erythrocytes of certain primate species $^{3}$. The release of HA from a parainfluenza virus (Sendai) by means of the combination of ether and a detergent (Emasol) was reported by Ноsaka,

* On sabbatical leave from the Department of Pathology, Tennis Court Road, Cambridge, England.

1 A. P. Waterson, J. G. Cruickshank, G. D. Laurence and A. D. Kaxarek, Virology 15, 379 [1961].

2 R. Rott and W. SснӓғER, Virology 14, 298 [1961].
Hosokawa and FukaI ${ }^{4}$. In view of these facts it seemed reasonable to investigate further the decomposition of the measles particle by this treatment. Such experiments might be expected to provide further evidence of similarity between the measles group and the parainfluenza group. The present paper deals with the preparation and properties of the components derived in this way from the measles virus, explains the morphological basis of their release from the particle, and also describes the practical usefulness of such a preparation.

\footnotetext{
3 J. R. Periés and C. Chany, C. R. hebd. Séances Acad. Sci. 251, 820 [1960].

4 Y. Hosaka, Y. Hosakawa and K. Fukai, Biken's J. 2, 367 [1959].
} 